\title{
X. - VALEUR ALIMENTAIRE CHEZ LE POULET DANS LES CONDITIONS PRATIQUES DE L'ÉLEVAGE
}

\author{
J. BARATOU et J.-P. VACHEL \\ Société Sanders \\ 17. Quai de l'Industrie, 91 - Juvisy-sur-Orge \\ RÉSUUMÉ
}

Dans des conditions pratiques d'alimentation, la valeur alimentaire des échantillons de maîs définis plus haut a été étudiée.

Bien qu'aucune đifférence de poids n'ait été statistiquement significative, certaines tendances se manifestent au niveau de l'interaction entre mode de préstockage et température de séchage.

Le séchage à $I_{4} 0^{\circ}$ après conservation par ventilation d'air refroidi apparaît le meilleur; le séchage à $80^{\circ}$ après échauffement contrôlé en cellule le moins bon.

\section{IN'TRODUC'TION}

Le maïs prend depuis quelques années une place grandissante dans l'industrie de l'alimentation animale, au détriment des céréales d'emploi traditionnel : avoine, orge et blé. Dans la plupart des cas le maïs doit être séché artificiellement. Le but de 1'expérimentation était de déterminer, parmi 6 traitements possibles du mais celui qui affecte le moins sa valeur alimentaire.

\section{MATÉRIEL E'T MÉTHODES}

\section{Les aliments composés}

On expérimente 21 aliments répartis en 7 gammes de 3 aliments, chaque gamme correspond à 2 répétitions de 7 lots d'animaux ( $\mathrm{I}$ lot témoin plus 6 lots expérimentaux correspondant aux 6 maïs référencés $R_{80}-R_{140}-E_{80}-E_{140}-F_{80}-F_{140}$ ). 
Chaque animal, depuis sa naissance jusqu'à son abattage, consomme 3 aliments au cours de sa croissance, selon les normes classiques actuelles :

\begin{tabular}{l|r}
\hline Période & Durée de distribution $(\mathrm{j})$ \\
\hline & \\
Démarrage & $0-23$ \\
Croissance & $23-35$ \\
Finition & $35-56$ \\
\hline
\end{tabular}

Pour mettre en évidence les conséquences des divers traitements subis par le maïs et une éventuelle variation de leur valeur nutritive, l'aliment témoin renferme un maĩs commercial de référence auquel on substitue dans les autres aliments les échantillons à tester.

La'composition et les caractéristiques calculées de ces aliments sont données dans le tableau $\mathbf{I}$.

TABLEAU I

Composition et caractéristiques calculées des aliments

\begin{tabular}{|c|c|c|c|}
\hline Formules distribuées de & 0 à $23 \mathrm{j}$ & 23 à $35 j$ & $35 \mathrm{à} 56 \mathrm{j}$ \\
\hline $\begin{array}{l}\text { Maïs } \ldots \ldots \ldots \ldots \ldots \ldots \ldots \\
\text { Tourteau de soja } 50 \ldots \ldots \ldots \ldots \ldots \\
\text { Suif } \ldots \ldots \ldots \ldots \ldots \ldots \\
\text { Composés minéraux pour poussins et poulets } \ldots \ldots\end{array}$ & $\begin{array}{c}62 \\
32 \\
3 \\
\text { q.s.p. } 100\end{array}$ & $\begin{array}{c}64 \\
29 \\
4 \\
\text { q.s.p. } 100\end{array}$ & $\begin{array}{c}69 \\
24 \\
4 \\
\text { q.s.p. } 100\end{array}$ \\
\hline 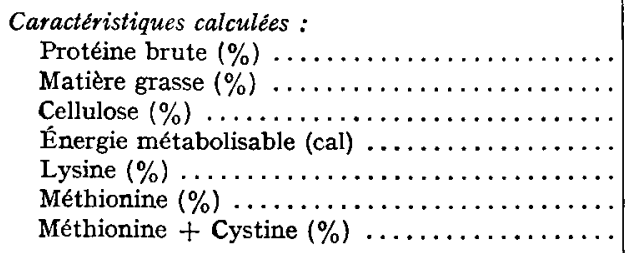 & $\begin{array}{l}22,2 \\
6,1 \\
2,7 \\
3157 \\
1,170 \\
0,465 \\
0,801\end{array}$ & $\begin{array}{l}20,9 \\
7,1 \\
2,7 \\
3217 \\
1,076 \\
0,480 \\
0,797\end{array}$ & $\begin{array}{c}18,9 \\
7,2 \\
2,6 \\
3265 \\
0,923 \\
0,454 \\
0,742\end{array}$ \\
\hline 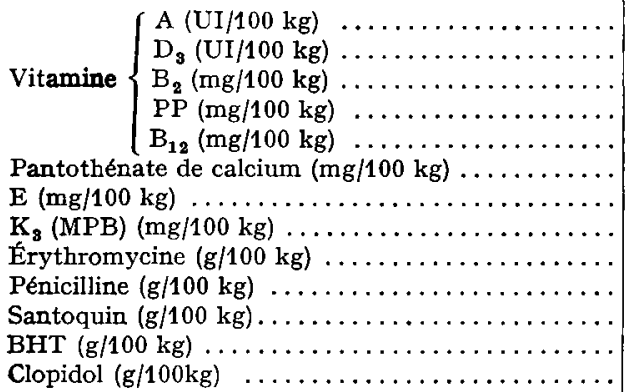 & $\begin{array}{c}7500000 \\
350000 \\
650 \\
2750 \\
1,50 \\
900 \\
1500 \\
550 \\
0,5 \\
1 \\
12,5 \\
0 \\
12,5\end{array}$ & $\begin{array}{c}6900000 \\
300000 \\
570 \\
2400 \\
1,50 \\
800 \\
400 \\
480 \\
0,5 \\
1 \\
- \\
12,5 \\
12,5\end{array}$ & $\begin{array}{c}6900000 \\
300000 \\
570 \\
2400 \\
1,50 \\
800 \\
400 \\
480 \\
0,5 \\
1 \\
- \\
12,5 \\
12,5\end{array}$ \\
\hline
\end{tabular}

Les animaux : leur répartition en lots

Quatre cent"quarante-huit poussins d'un jour, de souche Arbor Acres VT $\times$ AA50 (pattes jaunes) sont répartis au hasard en 14 lots de 32 (I 6 mâles et 16 femelles dans chaque lot). Chaque traitement est répété deux fois.

Les animaux sont élevés au sol selon les règles classiques de l'élevage. 


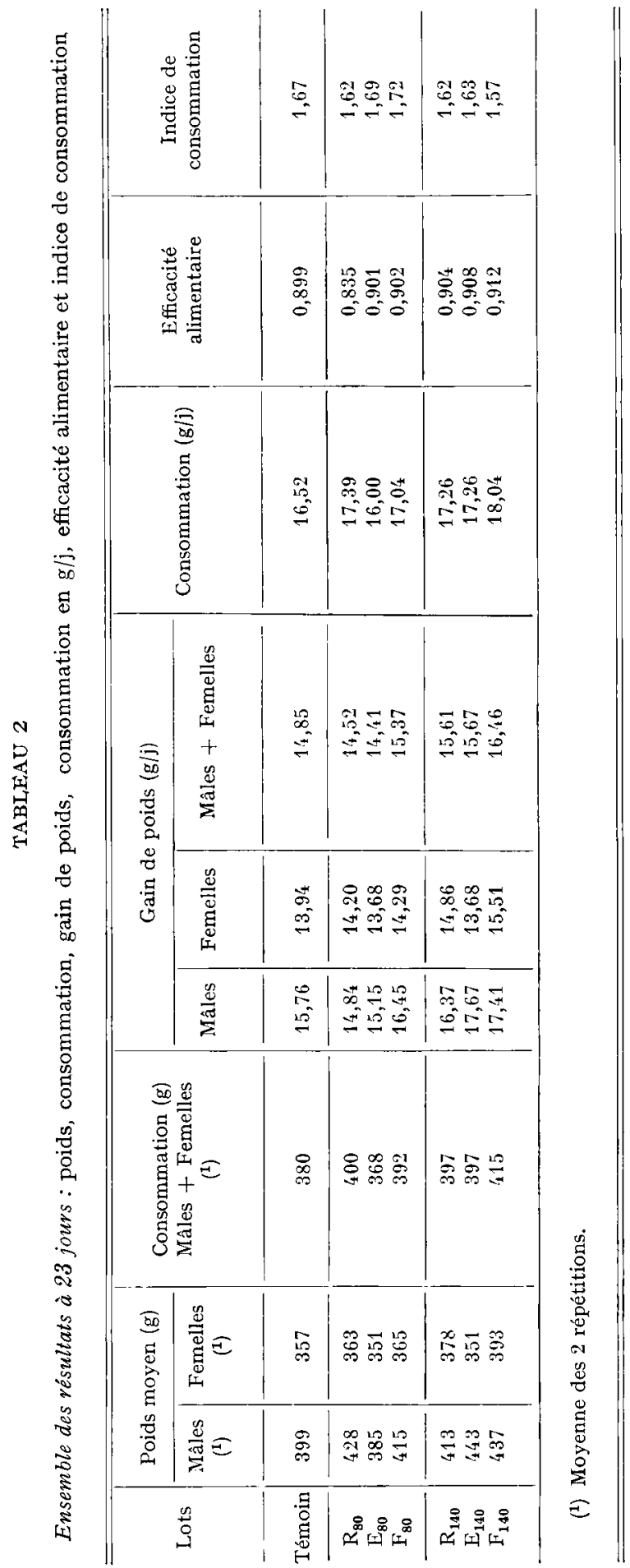


J. BARATOU, J.-P. VACHEL,

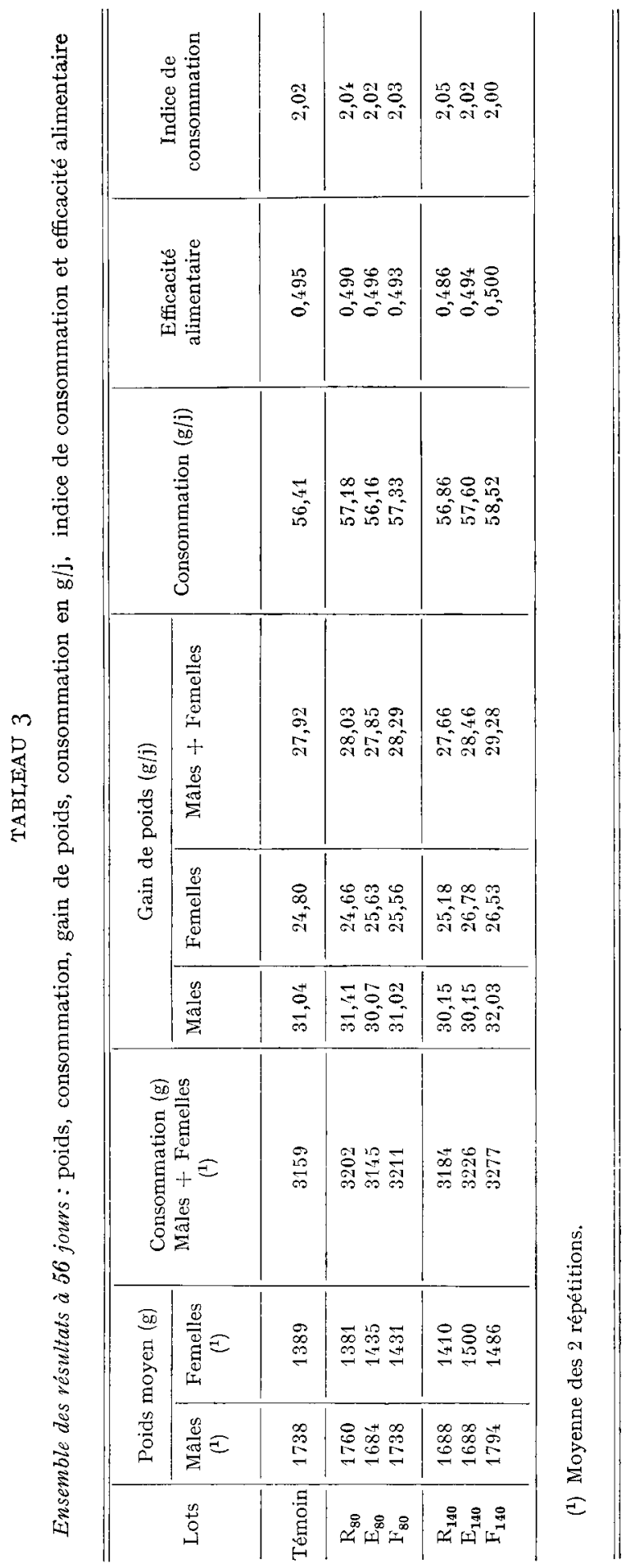




\section{Mesures enregistrées}

L'aliment est distribué ad libitum. On a enregistré le poids global des sujets et le poids global d'aliment consommé par un lot à 23,35 et 56 jours.

\section{RÉSULTATS}

Les résultats à 23 et à 56 jours :

- de poids et de consommation d'aliment,

- de gain de poids journalier,

- de consommation journalière,

- d'efficacité alimentaire et d'indice de consommation sont donnés dans les tableaux 2 et 3 .

L'analyse statistique des résultats ne nous a pas permis de noter de différence significative entre les traitements. Cela est vraisemblablement dû au faible nombre de répétition par régime ( 2 répétitions par régime).

Les autres tableaux permettent cependant de se faire une opinion sur les différences de valeur alimentaire de ces maïs.

Ainsi, le classement des maïs, d'après les poids et indices de consommation fait ressortir que :

I. Le maïs $\mathrm{F}_{140}$ (séché à $\mathrm{I}_{40^{\circ}}$ après conservation sous ventilation réfrigérée) est de meilleure qualité puisqu'il permet les meilleures performances dans presque tous les cas.

\section{TABLEAU 4}

Influence de la température de chauffage quel que soit le mode de conservation Pesée à 56 jours (poids moyen des deux répétitions)

\begin{tabular}{l|c|c}
\hline \multicolumn{1}{c}{ Nom du lot } & Mâles & Femelles \\
\hline & & \\
Témoin $\ldots \ldots \ldots \ldots \ldots \ldots$ & 1738 & 1389 \\
Maîs $80^{\circ} \ldots \ldots \ldots \ldots \ldots$ & 1727 & 1416 \\
Maïs $140^{\circ} \ldots \ldots \ldots \ldots \ldots$ & 1724 \\
\hline
\end{tabular}

2. Le maïs $\mathrm{E}_{8 n}$ (séché à $80^{\circ}$ après échauffement contrôlé en cellule) est celui dont la valeur nutritive a été la plus atteinte, car il donne souvent les plus mauvaises performances, surtout chez les mâles.

L'influence de la température : $80^{\circ} / 140^{\circ}$ quel que soit le mode de conservation apparaît dans le tableau 4 . On constate très peu de différence entre la moyenne des 3 lots " $80^{\circ}$ " et celle des 3 lots " r $40^{\circ}$ " surtout pour les mâles.

L'influence du traitement de conservation, quelle que soit la température apparaît dans le tableau 5. D'après l'ensemble des résultats de mâles + femelles, il semble que le meilleur traitement soit le séchage après conservation par ventilation d'air refroidi $(F)$. 
TABLEAU 5

Influence du traitement de conservation quelle que soit la température de traitement Pesée à 56 jours (poids moyen des deux répétitions)

\begin{tabular}{|c|c|c|}
\hline Nom du lot & Mâles & Femelles \\
\hline $\begin{array}{l}\text { Témoin } \ldots \ldots \ldots \ldots \ldots \ldots \ldots \ldots \\
\text { Séchage à la récolte }(\mathrm{R}) \ldots \ldots \ldots \ldots \\
\text { Séchage après échauffement contrôlé } \\
\quad \text { en cellule }(\mathrm{E}) \ldots \ldots \ldots \ldots \ldots \ldots \\
\text { Séchage après conservation sous } \\
\text { ventilation réfrigérée }(\mathrm{F}) \ldots \ldots \ldots\end{array}$ & $\begin{array}{l}1738 \\
1724 \\
1686 \\
1766\end{array}$ & $\begin{array}{l}1389 \\
1396 \\
1468 \\
1459\end{array}$ \\
\hline
\end{tabular}

\section{DISCUSSION ETT CONCLUSIONS}

Il était généralement admis que les traitements thermiques entraînaient une perte d'efficacité pour la croissance correspondant à une diminution des CUD des protides et de leur VB. Cependant, le chauffage n'agissait pas par une diminution des acides aminés, de la Lysine en particulier (JACQUOT, MATET et FridENSON, I947).

CALET et de LAMBILly (I960) CALET et TARDIF (I960) ont montré que lorsqu'on laisse s'établir une fermentation en stockant le grain $24 \mathrm{~h}$ avant le séchage, on assiste à une réduction de l'efficacité pour la croissance. L'échauffement du grain se traduit par une diminution de la disponibilité des acides aminés.

Dans l'essai présent, nous retrouvons des conclusions voisines. Pour une température de $80^{\circ}$ les mais qui n'ont pas eu la possibilité de fermenter (séchés immédiatement après la récolte et refroidis) ont une meilleure efficacité que ceux qui se sont échauffés en cellule. La température de $\mathrm{I}_{40^{\circ}}$ pour deux traitements sur trois (séchage immédiatement après la récolte et séchage après l'échauffement contrôlé en cellule) semble être trop élevée et causer la perte d'efficacité de croissance décrite depuis longtemps. L'exception du mais séché à $I 40^{\circ} \mathrm{C}$ après conservation par ventilation d'air refroidi peut peut-être s'expliquer par une température dans la masse du grain moins élevée. Le tableau $\mathrm{n}^{0} 3$ de LASSERAN (I) montre que la température de l'air d'attaque (colonne I) est de $140^{\circ}$ pour les trois échantillons, mais que l'air usé (colonne 2) a par rapport aux deux autres traitements une température inférieure pour le maiss conservé par ventilation froide. De même, le temps moyen de séchage (colonne 8 ) est nettement plus court que pour le maïs traité après la récolte (66 p. roo). Tout semble se passer comme si un traitement par air chaud à $140^{\circ}$ sur un grain ayant une température de $9^{\circ} \mathrm{C}$ a un effet moins néfaste qu'un traitement par air chaud à $140^{\circ} \mathrm{C}$ sur un grain ayant une température supérieure à $20^{\circ} \mathrm{C}$.

I1 est donc extrêmement important pour le fabricant d'aliment du bétail de disposer, pour contrôler a priori la qualité du maïs, de tests analytiques ou visuels qui soient non seulement simples et pratiques, mais également en corrélation la plus étroite possible avec les résultats zootechniques (croissance, indice de consommation). Notre contrôle macroscopique classique pour les céréales consiste essentiel- 
lement en un comptage des grains germés, cassés, moisis, brûlés, étrangers, etc., contrôle établi d'après les normes de qualité officielles diffusées chaque année par le décret de campagne.

Dans la présente expérience, ces critères se sont révélés inefficaces puisque sur les 6 échantillons, seul le maïs $R_{80}$ est " sain, loyal et marchand ".

\section{SUMMARY}

\section{X. - NUTRITIONAL, VALUE FOR CHICKENS UNDER PRACTICAL, CONDITIONS}

The nutritive value for chickens has been measured under the current practical conditions of the feed industry.

No statistical differences are observed under such conditions of large security according to the level in the commercial diet of all nutrients and especially that of amino acids. However, some tendencies appear from an interaction between condition of storage $\times$ drying temperature.

Drying at $14^{\circ}{ }^{\circ} \mathrm{C}$ after air-cooling appears to be the best treatment. On the contrary, drying at $80^{\circ} \mathrm{C}$, after spontaneous heating, is the worst.

\section{RÉFÉRENCES BIBLIOGRAPHIQUES}

Calet C., de Lambilly H., I960. Étude de la valeur alimentaire du maïs grain séché artificiellement pour le poussin en croissance. I. Influence du mode de séchage sur la disponibilité des acides aminés. Ann. Zootech., 9, I8r-184.

Calet C, TARDif H., ig6o. Étude de la valeur alimentaire du maîs grain séché artificiellement pour la croissance du poussin. II. Influence de la durée qui sépare la récolte du séchage. Ann. Zootech., $\mathbf{8}$, $35 \mathrm{I}-354$.

JACQuOT R., MATET J., FRIDENSON O., I947. Influence des traitements thermiques industriels sur la valeur protidique des aliments. Ann. Nutr. Alim., 1, I82-2r3. 